\title{
PADME - new code for modeling of planet georesources formation on heterogeneous computing systems
}

\author{
Viktor Protasov ${ }^{1, *}$, Igor Kulikov², Igor Chernykh ${ }^{2}$, and Irek Gubaydullin ${ }^{3}$ \\ ${ }^{1}$ Novosibirsk State Technical University, 630073 Novosibirsk, Russia \\ ${ }^{2}$ Institute of Computational Mathematics and Mathematical Geophysics SB RAS, 630090 \\ Novosibirsk, Russia \\ ${ }^{3}$ Institute of Petrochemistry and Catalysis RAS, 450075 Ufa, Russia
}

\begin{abstract}
Many planets were detected in last few years, but there is no clear understanding of how they are formed. The fairly clear understanding of Solar system formation was founded with time, but there are some doubts yet because we don't know what was at the beginning of the process, and what was acquired afterward. Moreover, formed ideas often couldn't explain some features of other systems. Searching for Earth-like terrestrial planets is another very important problem. Even if any of found exoplanets will be similar to Earth, we couldn't say that it is a "second Earth" exactly because its internal, geological, composition could be different - Venus is a vivid example. A new method for modelling of the planet formation process in a $3 \mathrm{D} 2 \mathrm{~V}$ formulation based on two-phase approach is presented in the paper. Fluids-in-cells method by Belotserkovskii-Davydov, modified with using the Godunov's scheme, is used to model the gas component. The dust component is described by Nbody system solved with the Particle-Mesh method. The method was accelerated by using of Nvidia CUDA technology. Gas-dust disk modelling results with the formation of sealing of gas and dust that could be interpreted as potential exoplanet are given.
\end{abstract}

\section{Introduction}

The search of planetary systems is one of the most important tasks of modern astronomy. In the beginning of $90^{\text {th }}$ with the advent of powerful telescopes, many extrasolar planets were discovered. At the end of 2014, its number was about 1800, and at the end of 2017 - more than 3700. The modern views of Solar system formation are based on KantLaplace theory, according to which the Sun and the planets were formed at the same time from circumstellar gas and dust. It describes the structure of the Solar system well enough but fails on some other systems. For example, hot Jupiters revolving too close to a star were discovered. Also, questions about the time needed to form the planetary system, and planet formation in multiple systems, are opened [1]. Researching of geophysical features of exoplanets becomes more popular [2] because the understanding of such properties could

\footnotetext{
${ }^{*}$ Corresponding author: pro.vik@bk.ru
} 
help not only in searching for Earth-like planets but will allow us to better understand processes inside planets.

There are two main approaches for modelling protoplanetary disks for today - twocomponent approach [3] and two-phase approach [4, 5]. The second one is used in the paper. To model a gas-component of a disk the Fluids-in-Cells method by BelotserkovskiiDavydov [6], modified with using the Godunov's scheme [7], is applied. Dust particles are described with N-body system which solved by the Particle-Mesh method [8]. To compute the gravitational interaction between gas and particles the Poisson equation for gravitational potential is solved by using the Fast Fourier Transform.

\section{Numerical method description}

The model of dynamics of a protoplanetary disk in cartesian coordinates is described by the following system of equations:

$$
\begin{gathered}
\frac{\partial \rho}{\partial t}+\operatorname{div}(\rho \mathbf{v})=0 \\
\frac{\partial \rho \mathbf{v}}{\partial t}+\operatorname{div}(\mathbf{v} \rho \mathbf{v})=-\operatorname{grad}(p)-\rho \operatorname{grad}(\Phi) \\
\frac{\partial \rho \varepsilon}{\partial t}+\operatorname{div}(\rho \varepsilon \mathbf{v})=-(\gamma-1) \rho \varepsilon \operatorname{div}(\mathbf{v}) \\
\frac{\partial \rho E}{\partial t}+\operatorname{div}(\rho E \mathbf{v})=-\operatorname{div}(p \mathbf{v})-(\operatorname{grad}(\Phi), \mathbf{v}) \\
\operatorname{div}(\operatorname{grad}(\Phi))=4 \pi G \tilde{\rho} \\
p=(\gamma-1) \rho \varepsilon \\
\rho E=\rho \varepsilon+\frac{\rho \mathbf{v}^{2}}{2} \\
\frac{d^{2} \tilde{\mathbf{x}}}{d t^{2}}=\frac{\mathbf{F}}{\tilde{m}} \\
\mathbf{F}=-\operatorname{grad}(\Phi)
\end{gathered}
$$

Equations (1) - (7) describes the dynamics of self-gravitating gas, and equations (8) (9) - dynamics of any particle. Here $\rho$ - gas density, $\mathbf{v}-$ speed of gas, $p$ - gas pressure, $\varepsilon-$ internal energy of the gas, $E$ - full energy of the gas, $\gamma$-adiabatic power, $\Phi$ - gravitational potential, $G$ - gravitational constant, $\tilde{\rho}$ - density of gas and particles distribution in $3 \mathrm{D}, \tilde{\mathbf{x}}-$ position of some particle, $\widetilde{m}-$ its mass.

\subsection{Gas component modelling}

The gas component is modelled using the Fluids-in-Cells method by BelotserkovskiiDavydov. It is based on the scheme of the splitting of physical processes - the solution of initial system of equations is divided into two stages. At the first, Euler, stage the gas dynamics system of equations is solved without advective members - recomputation of parameters is done in assumption that gas is still and has constant density. For a rectangular grid, the approximation is as follows:

$$
\frac{\rho_{i, j}^{n+1}-\rho_{i, j}^{n}}{\Delta t}=0
$$




$$
\begin{gathered}
\frac{\rho v_{x i, j}^{n}-\rho v_{x i, j}^{n-1}}{\Delta t}=-\frac{p_{i+1, j}^{*}-p_{i-1, j}^{*}}{h_{x}}-\rho_{i, j}^{n-1} \frac{\Phi_{i+1, j}^{n}-\Phi_{i-1, j}^{n}}{2 h_{x}} \\
\frac{\rho v_{y_{i, j}}^{n}-\rho v_{y_{i, j}}^{n-1}}{\Delta t}=-\frac{p_{i, j+1}^{*}-p_{i, j-1}^{*}}{h_{y}}-\rho_{i, j}^{n-1} \frac{\Phi_{i, j+1}^{n}-\Phi_{i, j+1}^{n}}{2 h_{y}} \\
\frac{p_{i, j}^{n}-p_{i, j}^{n-1}}{\Delta t}=-(\gamma-1) p_{i, j}^{n-1}\left(\frac{v_{x i+1, j}^{*}-v_{x i-1, j}^{*}}{h_{x}}+\frac{v_{y_{i, j+1}}^{*}-v_{y_{i, j-1}}^{*}}{h_{y}}\right)
\end{gathered}
$$

Equations (11) and (12) is obtained from the equation (2) and allows to compute $\mathrm{x}$ - and $\mathrm{y}$-components of impulse, respectively. The equation for pressure (13) is obtained from (3) with consideration of (6).

The second stage - Lagrangian. At this stage the advection of the gas is done, all the divergence operators that were not taken into account at the Euler stage are approximated. In simple terms, for each cell, we should compute how much of the gas flowed into it, how much flowed out and calculate the balance:

$$
M_{i, j}^{n+1}=M_{i, j}^{n}+\frac{\Delta t}{h_{x}}\left(x_{-} \text {flow }_{-} i n_{i, j}-x_{-} \text {flow }_{-} \text {out } t_{i, j}\right)+\frac{\Delta t}{h_{y}}\left(y_{-} \text {flow_in }_{i, j}-y_{-} \text {flow }_{-} \text {out } t_{i, j}\right) .(14
$$

To compute the flows through the borders of cells we use the modification of classical approach that considers possible "skew" of the border due to different velocities of the gas in nodes of a cell:

$$
x_{-} \text {flow_out }_{i, j}=\frac{1}{2} \sum_{k=0}^{1} v_{x_{i+1 / 2, j+(k-1 / 2)}}\left\{\begin{array}{c}
M_{i, j}, v_{x_{i+1 / 2, j+(k-1 / 2)}}>0 \\
M_{i+1, j}, v_{x_{i+1 / 2, j+(k-1 / 2)}} \leq 0
\end{array}\right.
$$

\subsection{Dust component modelling}

The main difficulty in modelling of the particles is in computing of the gravitational force affecting any particles from the others, and the necessity of accounting the interaction between gravitational fields of gas and particles. In the paper, the Particle-Mesh method, which allows reducing the computational costs at this stage of simulation, is used.

The main idea of the method lies in the fact that we compute interaction between particles not as "each-to-each", but projecting them into the similar rectangular grid as used for the gas component and computing the density of particles in each cell with consideration of their masses. Then the Poisson equation for the gravitational potential is solved for that distribution of density. Knowing the potential we could compute the gravitational force easily as $\mathbf{F}=-\operatorname{grad}(\Phi)$, where $\Phi$ is computed from (5) with consideration that $\tilde{\rho}=\rho_{\text {gas }}+\rho_{\text {particles }}$.

Such approach allows naturally consider the gravitational interaction between gas and particles, but its accuracy is low. The density of a particle in cell and force affecting it are computed by Clouds-in-Cells method [9] to reduce the error of computations. In this method, the particle coordinates are the coordinates of the center of mass of a "cloud" with finite size. The density of that cloud is distributed among cells into which it fells:

$$
\begin{gathered}
\rho_{i, j}=\sum_{c \in \text { clouds }} W_{i, j}(x, y) \rho_{c}(x, y) \\
\mathbf{F}_{c}=-\sum_{i, j} W_{i, j}(x, y) \operatorname{grad}(\Phi)_{i, j} \\
W_{i, j}(x, y)=\left\{\begin{array}{c}
\left(1-\frac{\left|x-x_{i}\right|}{h_{x}}\right)\left(1-\frac{\left|y-y_{j}\right|}{h_{y}}\right),\left|x-x_{i}\right| \leq h_{x} \& \&\left|y-y_{j}\right| \leq h_{y} \\
0, \text { else }
\end{array}\right.
\end{gathered}
$$


This approach doesn't completely solve the problem, of course, but it significantly reduces the error of computations as shown in [9].

\section{Gas-dust disk modelling}

Let's consider the region $[-2.5 ; 2.5] \times[-2.5 ; 2.5]$, in which is the gas with parameters:

$$
\rho(r)=\left\{\begin{array}{c}
2, r<2 \\
3, r<0.25
\end{array}, p(r)=\left\{\begin{array}{c}
1, r<2 \\
2, r<0.25
\end{array}, \omega(r)=2(2-r)^{2}\right.\right.
$$

Also in region $r \in[0.5 ; 2.0]$ uniformly distributed 50000 of particles with mass $m=5 \cdot 10^{-5}$ and angular velocity $\omega(r)=2(2-r)^{2}+\varepsilon$, where $\varepsilon-$ is a tiny deviation.

The formation of the gas-dust ring is shown in figure 1 . The seal consisting mainly of particles is formed inside of it. This sealing could be interpreted as a potential exoplanet.

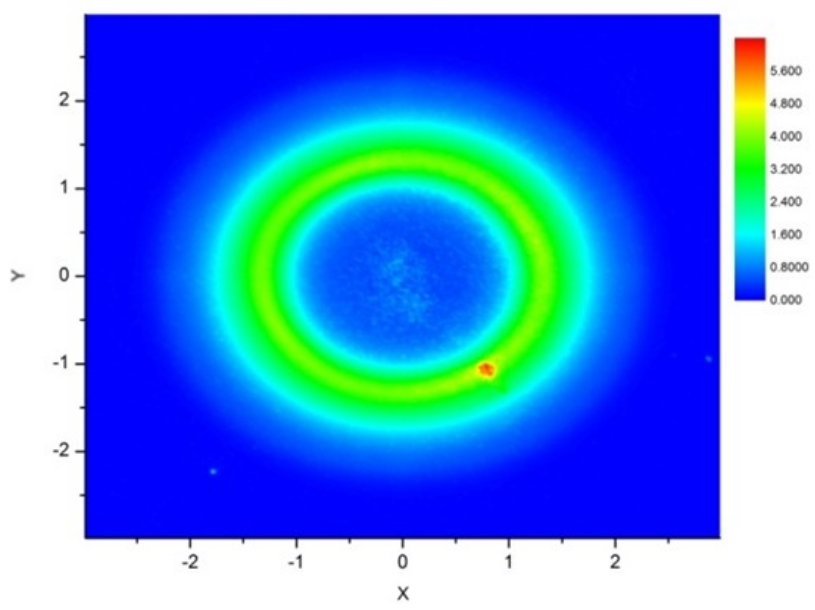

Fig 1. Modeling of gas-dust disk, $\mathrm{t}=0.4$.

\section{Discussion}

The described method is not a final point. It is just a timid step on the road to high-quality algorithms of numerical astrophysics which allows answering the most important questions about the Universe highly accurate. At the moment, active work is underway to improve the method. For example, it is possible to sufficiently improve the accuracy of computations if the magnetic field of the gas-dust disk will be taken into account. Yet another way is to recompute the grid accordingly to the medium parameters. In addition, for an adequate modelling of planetary cores, it is needed to consider the interaction between gas and particles not only by gravitational force but by collisions, adhesion, different chemical processes and transition of a substance from one state to another.

\section{Conclusions}

A new method for modelling of the planet formation process based on the Fluids-in-Cells method and the Particle-Mesh method oriented for using in heterogeneous computing systems is given in the paper. The gas component is modelled by Fluids-in-Cells method by Belotserkovskii-Davydov, modified with using the Godunov's scheme, is used to model the gas component. The dust component is described by N-body system solved with the 
Particle-Mesh method. Gravitational interaction between gas and particles is computed by solving the Poisson equation for the gravitational potential with Fast Fourier Transform. Gas-dust disk modelling results with the formation of sealing of gas and dust that could be interpreted as potential exoplanet are given.

The research work was supported by the Grant of the President of Russian Federation for the support of young scientists MK-1445.2017.9, RFBR grants 18-01-00166, 18-07-00757, and 17-31-50023.

\section{References}

1. A. Tutukov, A. Fedorova, Astron. Rep. 54, 305 (2012)

2. C. Dorn et.al., A\&A 577, 83 (2015)

3. S. Inaba et al., A\&A 431, 365 (2005)

4. D. Hubber et al., A\&A 529, (2011)

5. V. Vshivkov et al., Nauchnyj vestnik NGTU 44, 69 (2011)

6. O. Belotserkovskii, Iu. Davydov, Metod krupnykh chastits v gazovoi dinamike (M.: Nauka, 1982)

7. A. Kulikovskii, N. Pogorelov, A. Semenov, Matematicheskie voprosy chislennogo resheniia giperbolicheskikh sistem uravnenii (M.: Fizmatlit, 2001)

8. R. Hockney, J. Eastwood, Computer Simulation Using Particles (New York: Adam Hilger, IOP Publication Ltd, 1989)

9. C. Birdsall, D. Fuss, J. Comput. Phys. 135, 141 (1997) 